\title{
Therapie und Prophylaxe von Dekubitalulzera - Teil 2
}

\author{
M. Schempf ${ }^{1}$, C. Warda ${ }^{2}$, M. Mentzel' ${ }^{3}$, Y.-B. Kalke ${ }^{4}$, K. Huch ${ }^{4}$ \\ 1 Nova Clinic Biberach an der Riß \\ 2 Donau-Ries Klinik Donauwörth \\ 3 Universitätsklinikum Ulm, Klinik für Unfall-, Hand-, Plastische und Wiederherstellungschirurgie \\ 4 Orthopädische Universitätsklinik Ulm am RKU
}

Die operative Therapie bedarf ebenfalls einer gründlichen Vorbereitung und der Behandlung von Begleiterkrankungen, die patienten- und problemorientiert ausgerichtet sein sollte. Das radikale chirurgische Débridement, wenn möglich in der „Pseudotumor-Technik“ nach Guttmann, steht an erster Stelle. Prinzipiell sollten dabei das Spenderareal und die versorgenden Gefäße geschützt werden. Der Entscheid der operativen Versorgung sollte nach einem Stufenkonzept der plastisch-chirurgischen Deckungsverfahren getroffen werden. Beim Ersteingriff sollte die eventuelle erforderliche Behandlung von Rezidiven mit eingeplant werden.

Im Regelfall ist ein zweizeitiges Vorgehen indiziert:

1. Gründliches operatives Wunddébridement zur Erzielung eines makroskopisch sauberen Wundgrundes.

2. Nach nochmaligem Ausschneiden der Defektfläche folgt die definitive Defektdeckung mit plastisch-chirurgischen Operationsverfahren.

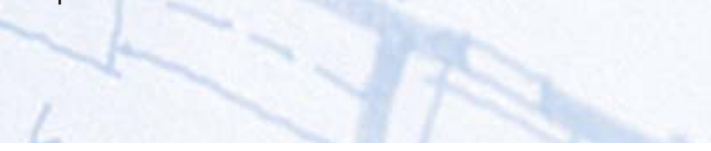

\section{Tabelle 1}

Operationsindikationen.

\section{Indikationen und Kontraindikationen zur konservativen vs. operativen Therapie}

Die Indikation zum operativen Verschluss ist von vielen Parametern abhängig. Es gibt hierzu vitale, absolute und relative Indikationen (Tab. 1). Abhängig von der Ausdehnung eines Dekubitus sollte primär ein konservativer Therapieversuch unternommen werden. Die konservative Therapie ist den Stadien I und II (nach Seiler) vorbehalten. Alle höhergradigen Ulzerationen sollten plastischchirurgischen Lösungen zugeführt werden. Konservativ können diese in der Regel nur schwer bzw. kaum zum Verschluss gebracht werden. Meist bildet sich ein instabiles Narbenfeld, aus dem sich oft erneute Ulzerationen bilden, da sie den erneuten Druckbelastungen auf Dauer nicht standhalten. Trotzdem sollte die operative Indikation v.a. bei multimorbiden Patienten streng gestellt werden, da diese möglicherweise einen operativen Eingriff aus anästhesiologischer Sicht nicht überleben bzw. eine fehlende Wundheilung aufweisen könnten. Allerdings sollte bei frisch querschnittgelähmten oder polytraumatisierten Patienten die frühzeitige plastische Deckung angestrebt werden, denn nur dann kann mit einer suffizienten Frührehabilitation begonnen werden. vitale Indikation

- Sepsis

- Arrosionsblutung

absolute Indikation

- Osteomyelitis

- Narbenkarzinom

- Fistel (Vagina, Urethra, Rektum)

relative Indikation

- Dekubitus Grad III und IV

- Dekubitusschmerzen

- beschleunigte Rehabilitation

- aufwendige Pflege

\section{Aufklärung}

Die Patienten müssen über allgemeine und spezifische OP-Risiken sowie Komplikationen aufgeklärt werden. Wundheilungsstörungen treten in $15-30 \%$ der Fälle auf, da es sich um „septische“ Operationen handelt. 


\section{Chirurgisches Wunddébridement}

Hierbei wird mit dem scharfen Löffel und Skalpell das nekrotische Gewebe entfernt. Dies wird in einer Sitzung meist nicht gelingen, da die Wundränder sich erst nach einer Latenzzeit demarkieren. Beim Débridement sollte das Gewebe so ausführlich wie nötig und sowenig wie möglich reseziert werden, um einerseits den Defekt nicht unnötig auszuweiten und um andererseits eine ideale Wundkonditionierung zu erreichen. Erst nach dem Débridement zeigt sich das ganze Ausmaß des Defekts.

An ein ggf. mehrfaches chirurgisches Débridement schließt sich die Wundkonditionierung an. Bei kleineren Ulzera beinhaltet dies die täglichen, mehrfachen Verbandwechsel mit mit Ringer-Laktat-Lösung versetzten Kompressen. NaCl-Verbände sind zelltoxisch. Es werden in der Literatur Verbandwechsel alle 4-6 Stunden empfohlen, um eine gute Reinigung der Wunde zu gewährleisten und Infekte früh zu erkennen. Die lokale Applikation von Antibiotika sollte vermieden werden, die systemische Gabe setzt eine manifeste Infektion voraus. Bei größeren Defekten können nach Débridement Vakuumverbände (VAC) zum Einsatz kommen.

\section{VAC-Therapie}

Die VAC-Therapie ist ein invasives, aktives Wundverschlusssystem, das durch Vakuum die Wundgranulation unterstützt. Durch intermittierenden oder kontinuierlichen Sog von $125 \mathrm{mmHg}$ wird durch eine feuchte Wundreinigung eine kontinuierliche Drainage mit Reduktion des Wundödems und Verbesserung der Durchblutung mit Angiogenese erreicht, und ein Granulationsgewebe entsteht. Zudem wird eine Umgebungsverschmutzung verhindert. Des Weiteren kommt es zur Reduktion der

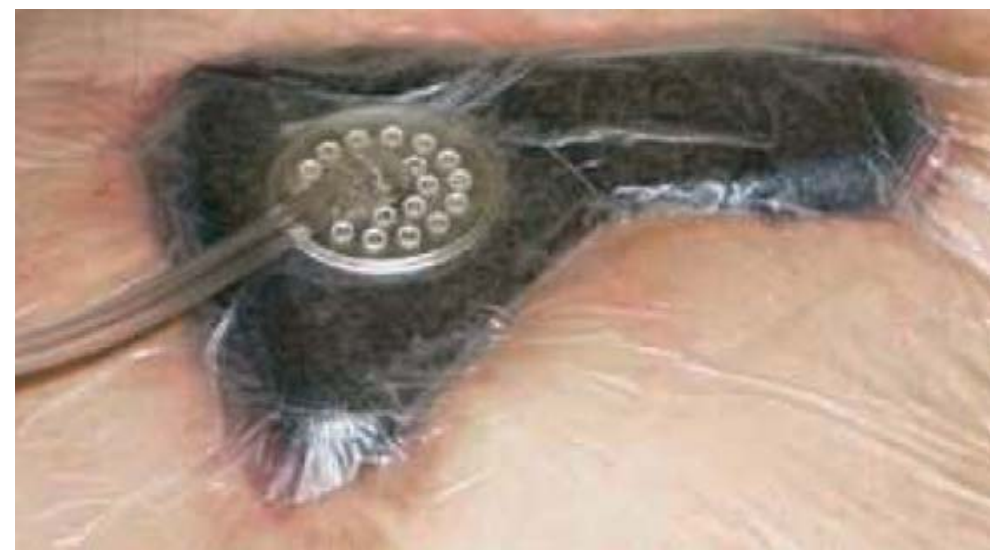

Abb. 1 - VAC-Therapie mit schwarzem Schwamm, dem Absaugansatz und einer Folienversiegelung. bakteriellen Kolonisationen und der Wundkontraktion durch Zugwirkung. Wird ein Vakuumsystem nicht richtig angewandt, kann ein zusätzlicher Druck auf das Ulkus entstehen (Abb. 1).

Eine komplette Wundheilung wird durch die VAC-Therapie nur selten erreicht. Erschwerend kommt eine wochenlange Behandlung hinzu, zudem ist die epithelialisierte Oberfläche wenig druckresistent. Chronische Wunden sollten somit einer zeitnahen Defektdeckung zugeführt werden. Jedoch kann die Indikation zum kompletten Wundverschluss mittels VAC-Dauertherapie gestellt werden, wobei dies auf wenige Patienten begrenzt bleiben sollte, die für eine chirurgische Defektdeckung nicht geeignet sind, wie z.B. Multimorbidität. Empfohlener Therapiemodus: $125 \mathrm{mmHg}$ mit Dauersog.

Nach Konditionierung der Weichteile sollte zügig eine Weichteildeckung mittels Lappenplastik oder in Einzelfällen durch Hauttransplantation erfolgen. Der VAC-Verband kann v.a. bei Hauttransplantationen als Fixierungshilfe auf das Transplantat gelegt werden. Durch die Sogwirkung wird eine gleichmäßige Fixierung des Transplantats auf der Wundoberfläche erreicht; dies dient der Serom- und Infektprophylaxe. Durch das feuchte Milieu wird die Nutrition des Transplantats erhöht.

Standard-Therapiemodus: $125 \mathrm{mmHg}$ kontinuierliches Vakuum, weißer PVA-Schwamm für 5-7 Tage (Abb. 2).

Komplexe, große Weichteildefekte können oft nicht mehr durch Lappenplastiken gedeckt werden. Hier kommt die „Delay“-Technik zum Einsatz. Hierbei kann ein Lappen in Etappen unter Einbeziehung von Angiosomen gehoben werden. Es wird der VAC dabei zwischen Lappen und Muskulatur platziert. Bei der sog. Präfabrikation können funktionelle Strukturen wie Haut, Knochen, Knorpel in den Lappen integriert werden.

Komplikationen bei VAC-Therapie werden in der Literatur selten beschrieben. Eine Komplikation stellt die Keimvermehrung v.a. bei Staphylokokkken (cave: MRSA) dar. Deshalb sollten die Systeme alle 3-5 Tage gewechselt werden. Weitere Komplikationen sind:

- Blutungen mit Anämie,

- Elektrolytstörungen,

- Hautmazeration,

- Schmerzen,

- Hypergranulation,

- Fibrosierung und Gelenkeinsteifung. 


\section{Prinzipien}

- Radikale Exzision des Ulkus mit Narbengewebe, Bursae und Weichteilverkalkungen.

- Bei Knochenbefall mit Osteitis sollte das Periost eröffnet und mit dem Meißel ca. 1 cm der Knochen abgetragen werden. Bei ausgeprägtem Befall des Knochens muss auch dieser débridiert werden. Eine Rekonstruktion von Knochenprominenzen zur besseren Druckverteilung kann erwogen werden.

- Zur Hämatomvermeidung Einlage mindestens einer Wunddrainage.

- Verschluss jedes potenziellen Totraums durch muskuläre oder deepithelisierte Lappenanteile.

- Planung von gut vaskularisierten Lappen, wobei die Nähte nicht in die druckbelastete Zone zu liegen kommen sollten.

- Verschluss des Hebedefekts durch spannungsfreie bzw. -arme Adaptation oder Hauttransplantate.

\section{Schwenklappenplastik}

Die Wahl des Operationsverfahrens zur plastischen Deckung wird von lokalen und allgemeinen Faktoren beeinflusst.

- Lokale Faktoren: Lokalisation des Ulkus, Größen und Tiefenausdehnung, Knochen und Gelenkbeteiligung, Haut und Weichteilzustand. Narben und Voroperationen können die Wahlmöglichkeiten der Lappen einschränken. Sorgfältige Planung der Lappenplastik, um die Gefäßversorgung anderer potenzieller Lappen für Rezidive zu erhalten.

- Allgemeine Faktoren: Diese hängt von EZ und AZ, der Compliance und psychosozialen Faktoren ab. Entscheidend ist die Rehabilitationsfähigkeit, wobei die Entscheidung, ob ein Patient in Zukunft vermehrt liegen oder sitzen wird, maßgeblich ist. Bei kachektischen Patienten kann ein muskulokutaner Lappen Vorteile bieten. Dagegen kann bei adipösen Patienten ein Kalibersprung des Subkutangewebes Probleme bereiten. Bei Paraplegikern mit Rückenmarkläsion distal L2/3 kann ein sensibler Tensor-fasciae-lataeLappen eine Reinnervierung von druckbelasteten Stellen bewirken. Bei Patienten mit stark reduzierter Compliance sollte erwogen werden, ob eine plastische Deckung Erfolg verspricht.

Eine Kombination von Débridement und sofortiger plastischer Deckung wird aus verschiedenen Gründen nicht empfohlen:
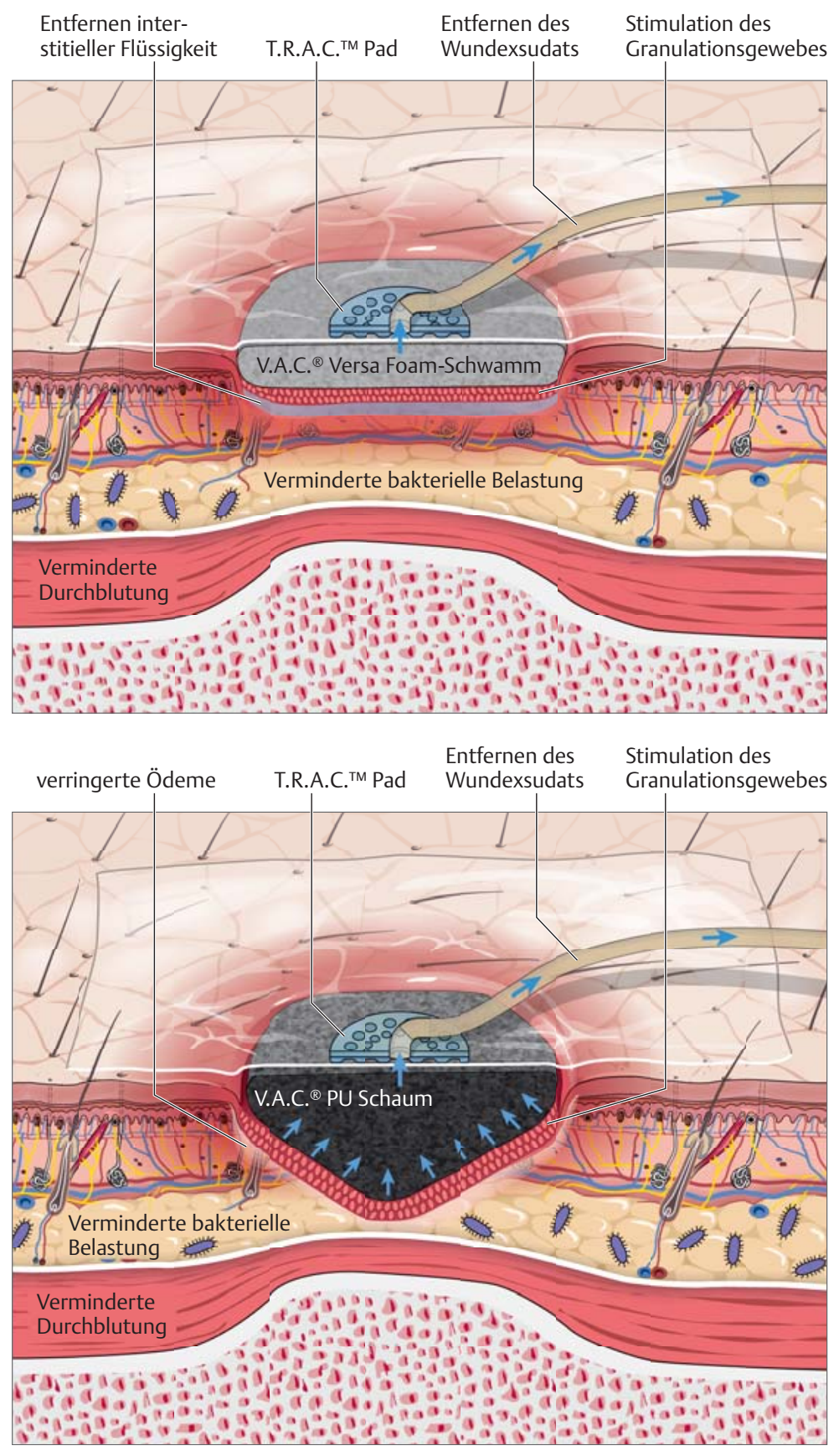

Abb. 2 - Grundprinzipien der unterschiedlichen Schwammanwendungen.

- Die Grenzen von gesundem und nekrotischem Gewebe sind nicht klar erkennbar. Damit entstehen Wundrandnekrosen bei primärer plastischer Deckung.

- Das Gewebe ist vom Druck noch geschädigt und enthält gewebetoxische Stoffe wie Proteasen und Zytokine.

- Vor dem Débridement kann eine erhebliche Bakterienlast vorhanden sein. Durch ein Débridement kann solche eine Verunreinigung in eine einfache Wundkontamination überführt werden. 
- Der Patient befindet sich ein einem reduzierten Allgemeinzustand. Durch 2-zeitiges Vorgehen konnte die Rezidivrate nach plastischer Deckung deutlich gesenkt werden.

Nach der Wundkonditionierung folgt das radikale Débridement in der Pseudotumor-Technik nach Guttmann. Dabei werden der Wundrand in einem ausreichenden Abstand umschnitten und die Wundränder mit Haltefäden miteinander einstülpend vernäht, sodass das Ulkus mit sämtlichem Granulationsgewebe in toto reseziert werden kann.

\section{- Lappenwahl}

Aufgrund der Komplexität der Schwenklappenchirurgie wird an dieser Stelle beispielhaft darauf eingegangen (v.a. im Beckenbereich), und wir verweisen hierzu auf detaillierte plastisch-chirurgische Literatur. Für die richtige Schwenklappenwahl sollten mehrere Kriterien in Betracht genommen werden:

- Muskellappen oder fasziokutane Lappen: In der Vergangenheit wurden überwiegend Muskellappen im Gegensatz zu den fasziokutanen Lappen durchgeführt. Inzwischen konnte allerdings in der Literatur eine weitgehende Ebenbürtigkeit der fasziokutanen Lappen gezeigt werden, da andere Faktoren, wie Lappenvolumen und Begleitosteitis eine ebenfalls entscheidende Rolle spielen.

- Sensible Lappen: Bei Querschnittgelähmten liegt es nahe, eine plastische Deckung mit sensibler Versorgung anzustreben, um Rezidive aufgrund Hypo- bzw. Asensibilität zu verhindern (z. B. TFL). Allerdings haben diese eine begrenzte Reichweite und einen Sensibilitätsverlust an der Donorstelle zur Folge.

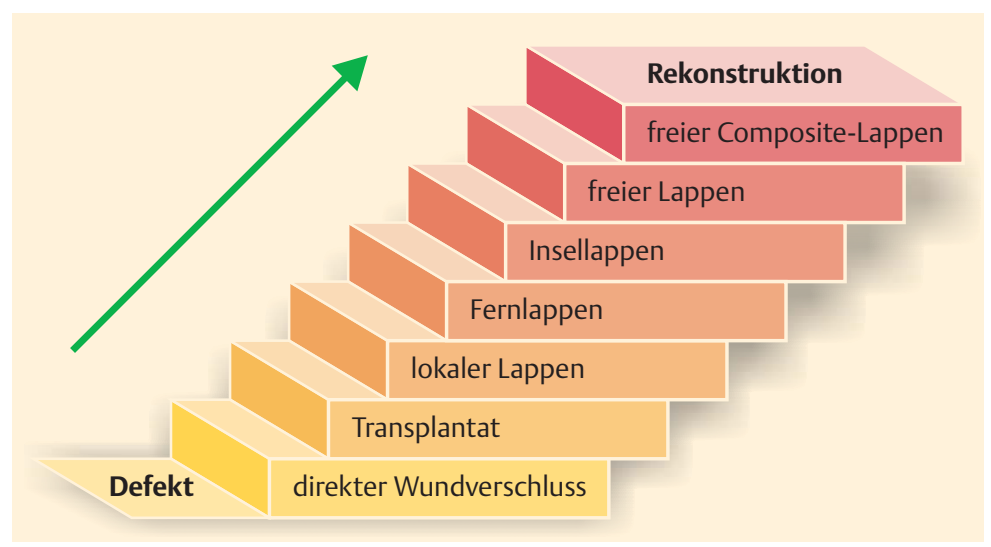

Abb. 3 - Rekonstruktive Leiter. Ein allgemeingültiges Stufenkonzept zur Defektdeckung in der plastisch-rekonstruktiven Chirurgie.
- Lappenplastik ohne definierte Gefäßversorgung: Dies sind Lappen aus unmittelbarer Umgebung des Defekts ohne definierte anatomische Gefäßversorgung, sog. Nahlappenplastiken („random pattern flaps“). Voraussetzung ist, dass die Lappen spannungsfrei im Defekt zu liegen kommen und die Hebestelle außerhalb der Belastungszone liegt. Hier kommt der meistbenutzte Limberg-Lappen zum Decken von kleinen Defekten im Bereich des Sakrums und der Sitzbeine zum Einsatz. Bei größeren Defekten eignet sich der fasziokutane Rotationslappen.

- Lappenplastik mit definierter Gefäßversorgung: Die Lappenplastiken mit definierter Gefäßversorgung beinhalten Muskellappen wie auch fasziokutane Lappen, die eine sehr gute Vaskularisation versprechen. Der fasziokutane Lappen zeigt sich druckresistenter als der Muskellappen, da der Muskel durch die Deinnervation atrophiert und fibrotisch umgebaut wird. Durch die Deinsertion an Ansatz oder Ursprung des Muskels kann es zu einer Kraftminderung kommen. Die Indikation für Muskellappen besteht für tiefe Defekte, bei denen Wundhöhlen ausgefüllt werden müssen. Nach fasziokutaner Lappendeckung besteht immer noch die Möglichkeit der Muskellappentransplantation bei Rezidiven.

- Freie mikrovaskuläre Lappenplastik: Bei jungen Patienten, z. B. rollstuhlfähige Paraplegiker, eignet sich die Deckung v.a. bei Osteomyelitis mit mikrovaskulären Lappen.

Prinzipiell sollte möglichst ein einzeitiger Verschluss angestrebt werden. Jedoch zeigt sich erst nach dem radikalen Débridement das gesamte Ausmaß des Defekts, sodass erst dann der Entscheid zur richtigen Lappenwahl gestellt werden kann. Zur Wahl des Lappen kann man sich am Stufenkonzept der rekonstruktiven Chirurgie (rekonstruktive Leiter) orientieren (Abb. 3). Dies richtet sich auch nach der anatomischen Region des Dekubitus und der Funktionalität des Schwenklappens (Tab. 2).

Primärverschluss. Der primäre Wundverschluss verbietet sich weitgehend, da es nach Verschließen der Haut meist zu Weichteilhöhlen kommt, die zu Hämatomen, Abszessen und Wunddehiszenzen neigen. Außerdem ist ein spannungsfreier Verschluss in schlecht durchblutetem Gewebe nicht möglich. Durch zugbedingte Wundrandischämie besteht die Gefahr der Vergrößerung des Dekubitus bzw. septischer Komplikationen. In Ausnahmefällen können sehr kleine Fisteln mit gutem Umgebungsgewebe oder einer prophylaktischen Kokzygektomie mit Primärverschluss behandelt werden. 
Tabelle 2

Lappenwahl nach anatomischer Region und Funktionalität (mod. nach Kremer et al. 1999).

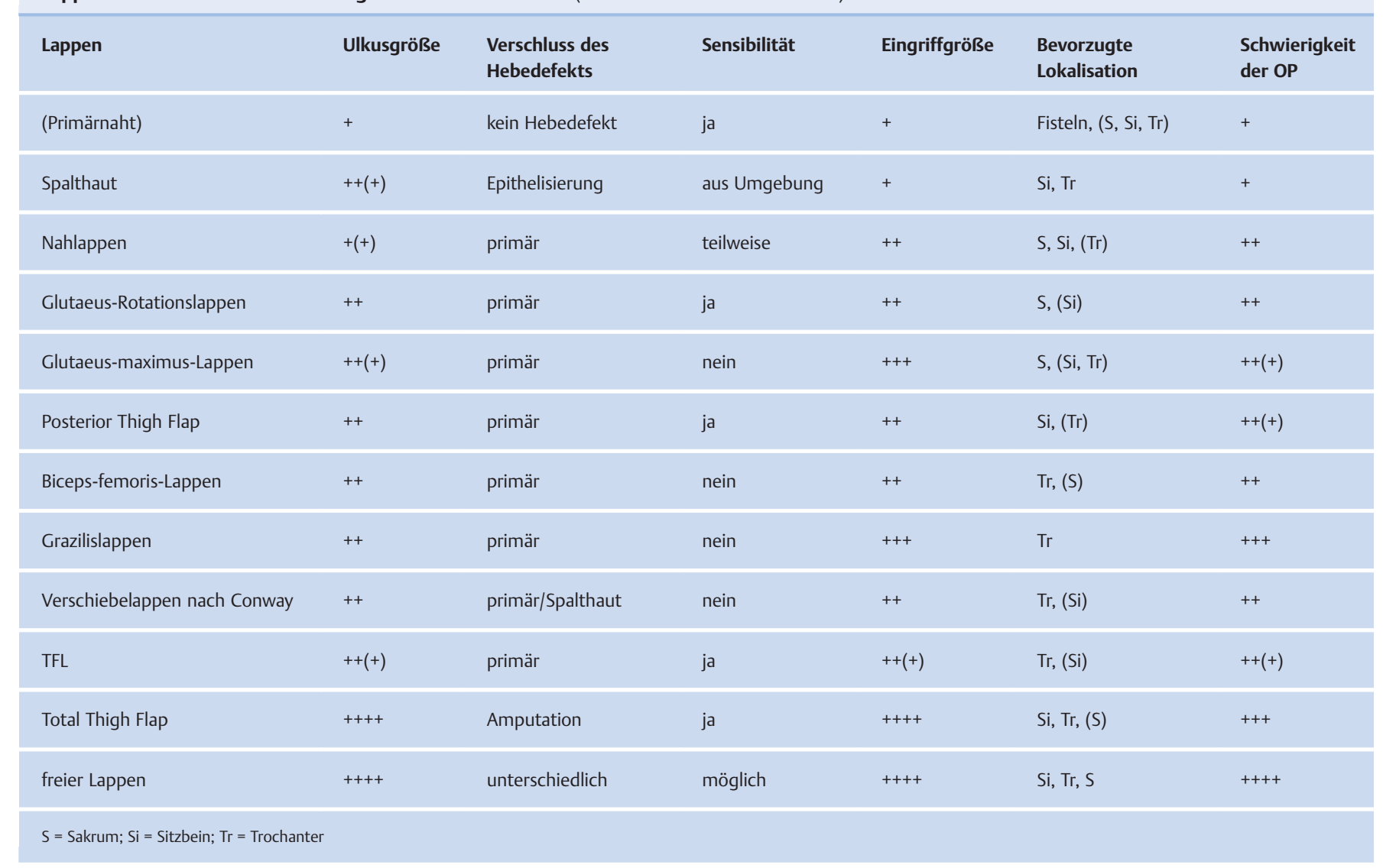

Spalthauttransplantation. Prinzipiell ist die Deckung eines Dekubitus mit Spalthaut nicht geeignet, da sie den Druckverhältnissen nicht standhalten kann und sich instabiles Narbengewebe bildet. Diese Therapie ist für Ausnahmesituationen vorbehalten, in denen sich Patienten in einem schlechten Allgemeinzustand befinden. Es setzt einen gut granulierten, keimarmen $\left(<10^{5} \mathrm{Keime} / \mathrm{g}\right.$ Gewebe) Wundgrund voraus. Die Überlebenschance der Hauttransplantation liegt in der Literatur bei 60\%. Mit dem Dermatom wird an geeigneter Stelle (z.B. Oberschenkel) ein 0,2-0,3 mm dickes Hauttransplantat entnommen. Eine Transplantatvergrößerung erreicht man durch die „Meshgraft-Technik“ (Gitternetz) die im Verhältnis $1: 1,5$ angefertigt werden sollte. Die Spalthaut wird geschützt durch Fettgaze und in Überknüpftechnik mit Schaumstoff oder Stahlwolle 5 Tage, bis zur vollständigen Vaskularisation, gesichert (alternativ: temporäre Vakuumversiegelung). Es schließt sich die Hautpflege mit Paraffinöl oder Panthenolsalbe an. Die Aufbelastung des Transplantats kann frühestens nach 21 Tagen begonnen werden.

\section{- Lappenwahl nach Dekubituslokalisation}

\section{Sakrumdekubitus}

Ulzera im Bereich des Sakrums treten v.a. bei respiratorischen und urogenitalen Infekten auf. Kleine Defekte können mit lokalen Hautlappen wie dem Rhomboidlappen nach Limberg oder Verschiebeschwenklappen nach Schrudde gedeckt werden (Abb. 4). Größere Defekte

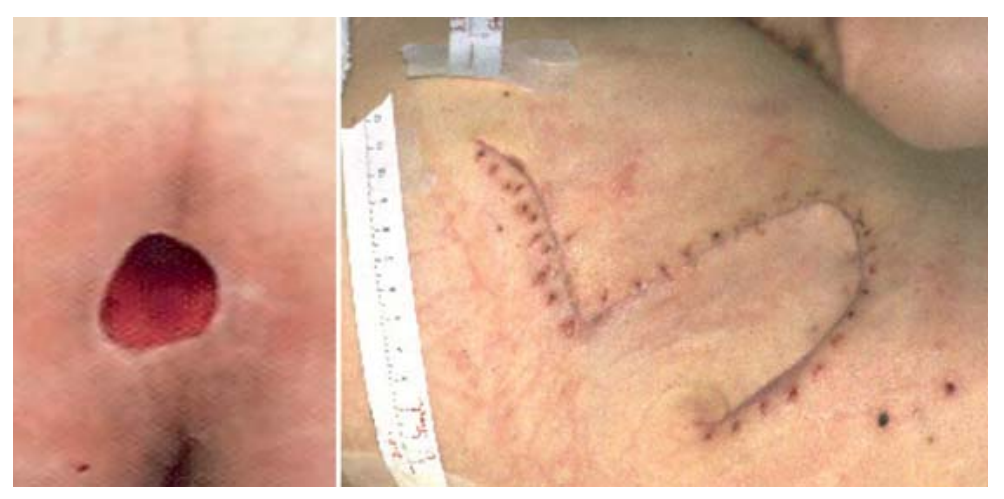

Abb. 4 - Sogenannter Schrudde-Lappen. 


\section{Grundlagen}

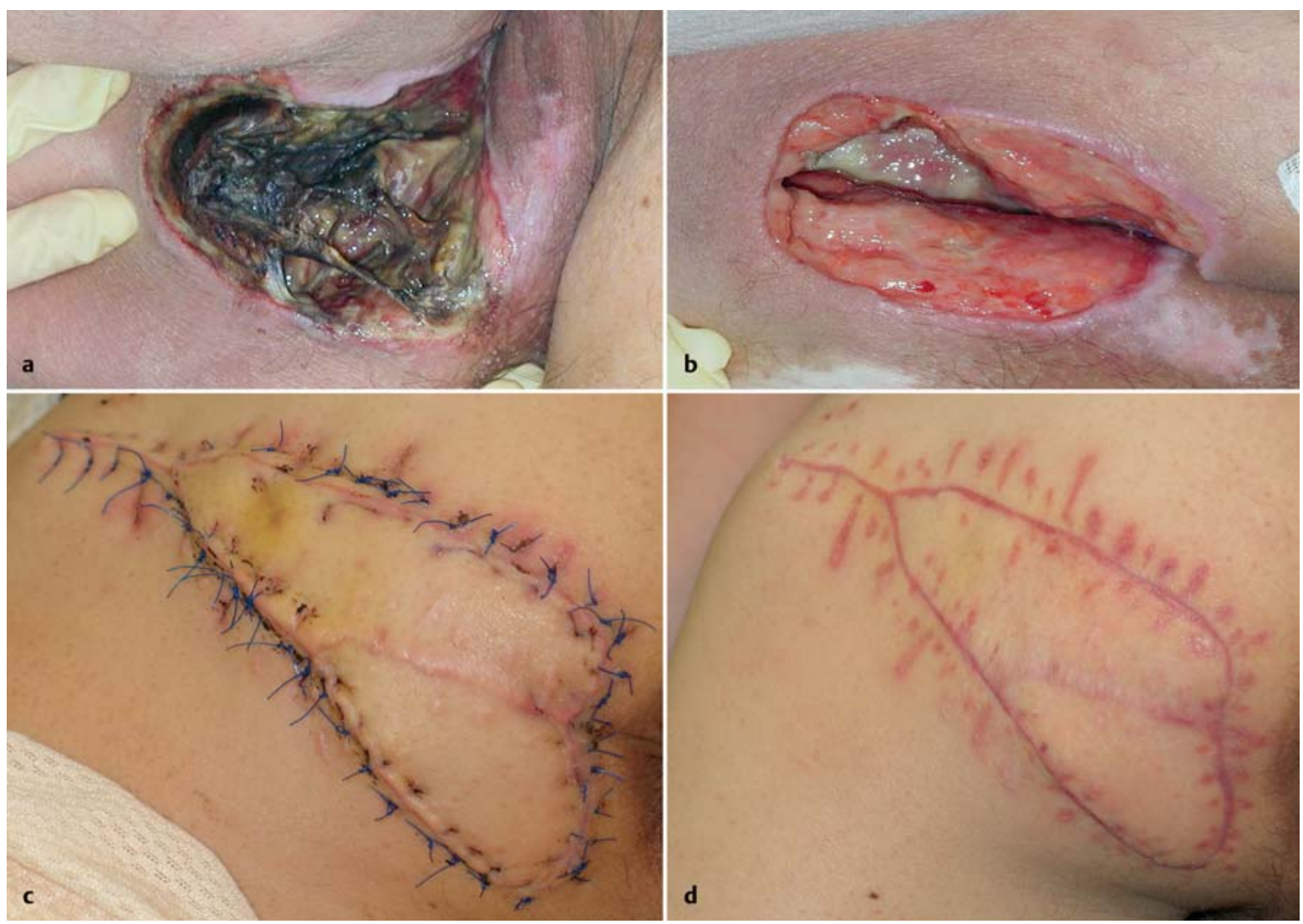

Abb. 5 - Fasziokutaner VY-Schwenklappen. a Primärer Dekubitus mit Nekrosen. b Z.n. Wundkonditionierung nach VAC-Anlage. c Fasziokutaner VY-Schwenklappen am 14. postoperativen Tag. d Ausheilung nach 6 Wochen.

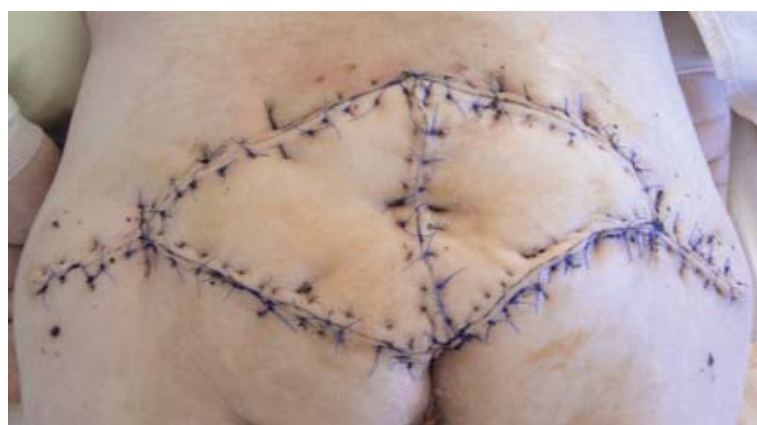

Abb. 6 - Fasziokutane doppelseitige VY-Plastik bei großem sakralem Defekt.

werden mit faszio- oder myokutanen Lappen der Glutäalregion aus dem Gefäßgebiet der oberen und unteren Glutäalarterien versorgt. Die Muskelursprünge und Ansätze an Sakrum, Crista iliaca und Tractus iliotibialis können durchtrennt werden, was die Mobilität des Lappens erhöht. Glutäallappen können als VY-Lappen (Abb. 5, Abb. 6), Rotationslappen (Abb. 7) oder Insellappen geschwenkt werden. Je größer der Lappen ist, umso mehr müssen die die Haut versorgenden Gefäße durchtrennt werden, was die Lappendurchblutung gefährden kann.

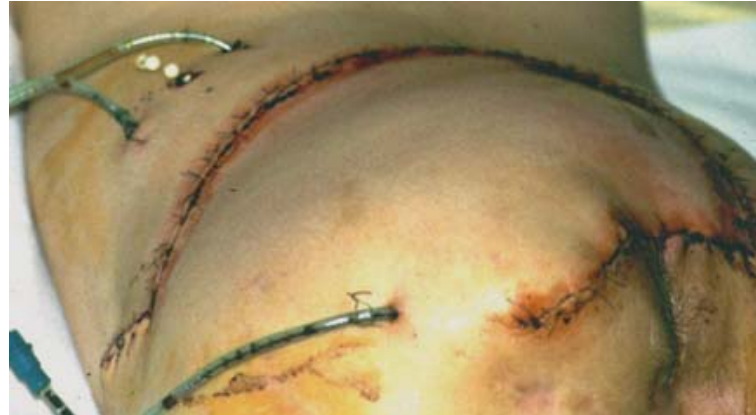

Abb. 7 - Rotationslappen zur Deckung eines sakralen Dekubitusulkus.

Eine Alternative stellt der Perforatorlappen dar. Hierbei werden mikrochirurgisch myokutane Perforatorgefäße präpariert, die ein definiertes Hautsegment (Angiosom) versorgen. Das Angiosom kann mit seinem Gefäßstiel über die Muskulatur gehoben werden. Der Vorteil dieser Methode ist die Schonung der Muskulatur, die Mobilität und dünne, flexible Hautinseln. 


\section{Tuber-ischiadicum-Dekubitus}

Ein optimaler Lappen zur Sitzbeindekubitusdeckung ist der fasziokutane „posterior thigh flap“, der durch die A. glutaea inferior und den $\mathrm{N}$. cutaneus femoris posterior versorgt wird.

Die Sitzbeinhöcker sollten ggf. geglättet werden, um eine gleichmäßige Druckverteilung der beiden Sitzbeine und dorsalen Oberschenkelregion zu erreichen. Von einer prophylaktischen Ischiektomie wird abgeraten, da sie eine hohe Inzidenz perinealer Ulzera und urogenitaler Komplikationen aufweist. Da in dieser Region gehäuft Rezidive eintreten, muss die Gefäßversorgung zukünftiger Lappenplastiken in die chirurgische Strategie einbezogen werden. Erste Wahl ist der „glutaeal thigh flap“ (Abb. 8), der die Gefäßversorgung anderer Lappen nicht gefährdet. Bei tiefen Ulzera kann hierbei der Biceps femoris mit eingeschlossen werden, wobei allerdings die inferioren Glutäalgefäße durchtrennt werden müssen. Alternativ kann der „inferior glutaeal artery perforator flap“ verwendet werden. Dieser gefäßgestielte Lappen bietet optimale Mobilität unter Schonung der inferioren Glutäalgefäße.

Ferner ist der Tensor-fasciae-latae-Lappen (TFL) zu erwähnen, der allerdings die dorsalen Gefäße der Oberschenkelregion verletzten kann (Abb. 9). Soll die Sensibilität durch das Versorgungsgebiet des N. femoris lateralis und $\mathrm{N}$. iliohypogastricus jedoch erhalten bleiben und geschwenkt werden, bietet der TFL einen Vorteil. Reservelappen ist der myokutane Grazilislappen.

\section{Trochanterdekubitus}

Typischerweise weisen Trochanterdekubitalulzera eher kleinere Hautdefekte auf, die oft mit Taschenbildung vergesellschaftet sind. Zur Trochanterdeckung bietet der „Tensor-fasciae-latae-Lappen“ (Abb. 9) durch eine gute Gefäßversorgung (A. circumflexa femoris lateralis) und durch Wiederherstellung der Sensibilität durch den $\mathrm{N}$. femoris cutaneus lateralis Vorteile.

Dieser kann als VY-Vorschublappen, Brückenlappen oder sensibler Lappen geschwenkt werden. Nachteilig kann es v. a. bei weiblichen und adipösen Patienten durch Auftragung des subkutanen Fettgewebes zur sog. „Dog-Ear“Bildung kommen. Alternativ wird in der lateralen Oberschenkelregion der „sliding flap“ verwendet. Als sehr schonende Variante kann der „anterolateral thigh flap“ benutzt werden.

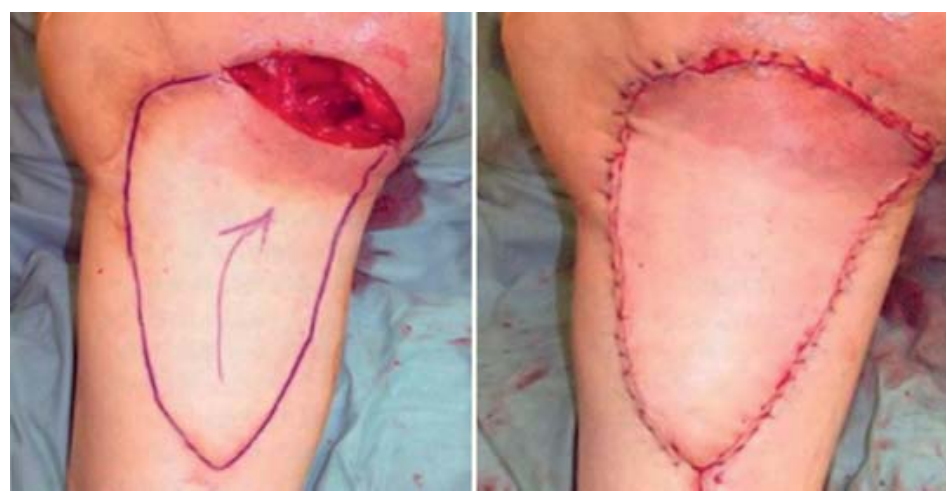

Abb. 8 - Glutaeal Thigh Flap in VY-Technik.

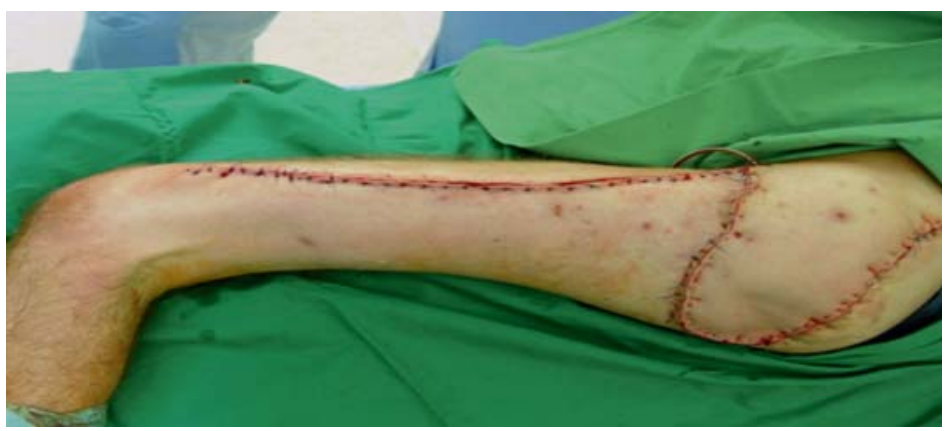

Abb. 9 - Tensor-fasciae-latae-Lappen mit primärem Verschluss des Entnahmebereichs.

\section{Perioperatives Management}

- Dekubitalulzera gelten primär als kontaminiert, da sie oft lange Zeit offen therapiert werden und oft in der Beckenregion lokalisiert sind. Dabei ist von einer Mischflora auszugehen, da Hautkeime mit Keimen aus dem urogenitalen System und Darmkeimen sich vermischen. Bei sauber granulierten Ulzera finden sich häufig Keimbesiedelungen mit Staphylokokken, E. coli, und Enterokokken, bei reduzierter Heilungstendenz Pseudomonas aeruginosa und Anaerobier.

- Die bakterielle Kontamination kann häufig nicht vollständig beseitigt, aber deutlich reduziert werden. In diesem Stadium ist eine plastische Deckung indiziert. Durch die Lappenplastik wird ein gut durchblutetes Gewebe in das Ulkus geschwenkt, womit eine verbesserte Gewebetrophik und Wirksamkeit von Antibiotika erzielt werden kann.

- Bei offener Wundbehandlung ist keine generalisierte antibiotische Therapie indiziert, außer bei entzündeten, phlegmonösen Ulzera oder septischen Krankheitsbildern. Prinzipiell erfolgt die antibiotische Therapie nach Antibiogramm, jedoch kann im Akutstadium eine Kombination eines Cephalosporins der 2. Generation mit Metronidazol verabreicht werden. 
- Ist eine definitive plastische Deckung geplant, sollte peri- und postoperativ eine resistenzgerechte Antibiotikagabe erfolgen, da diese Operationen Wundheilungsstörungen in bis zu 20-35\% der Fälle aufweisen. Es kann intraoperativ über eine Einlage eines Antibiotikaträgers entschieden werden. Postoperativ sollte bei Lappenplastiken mindestens 5-7 Tage, bei Osteomyelitis mindestens 6 Wochen eine antibiotische Therapie erfolgen. Die Drainage kann 5-10 Tage belassen werden.

- Zur Prophylaxe thromboembolischer Komplikationen sollte eine Thromboseprophylaxe mit niedermolekularem Heparin perioperativ eingeleitet werden.

- In der Literatur wird eine präoperative Darmreinigung empfohlen, da hierdurch das Infektionsrisiko um $30 \%$ gesenkt werden kann.

- Die Urinableitung kann durch Dauerkatheter oder suprapubische Harnableitung erfolgen.
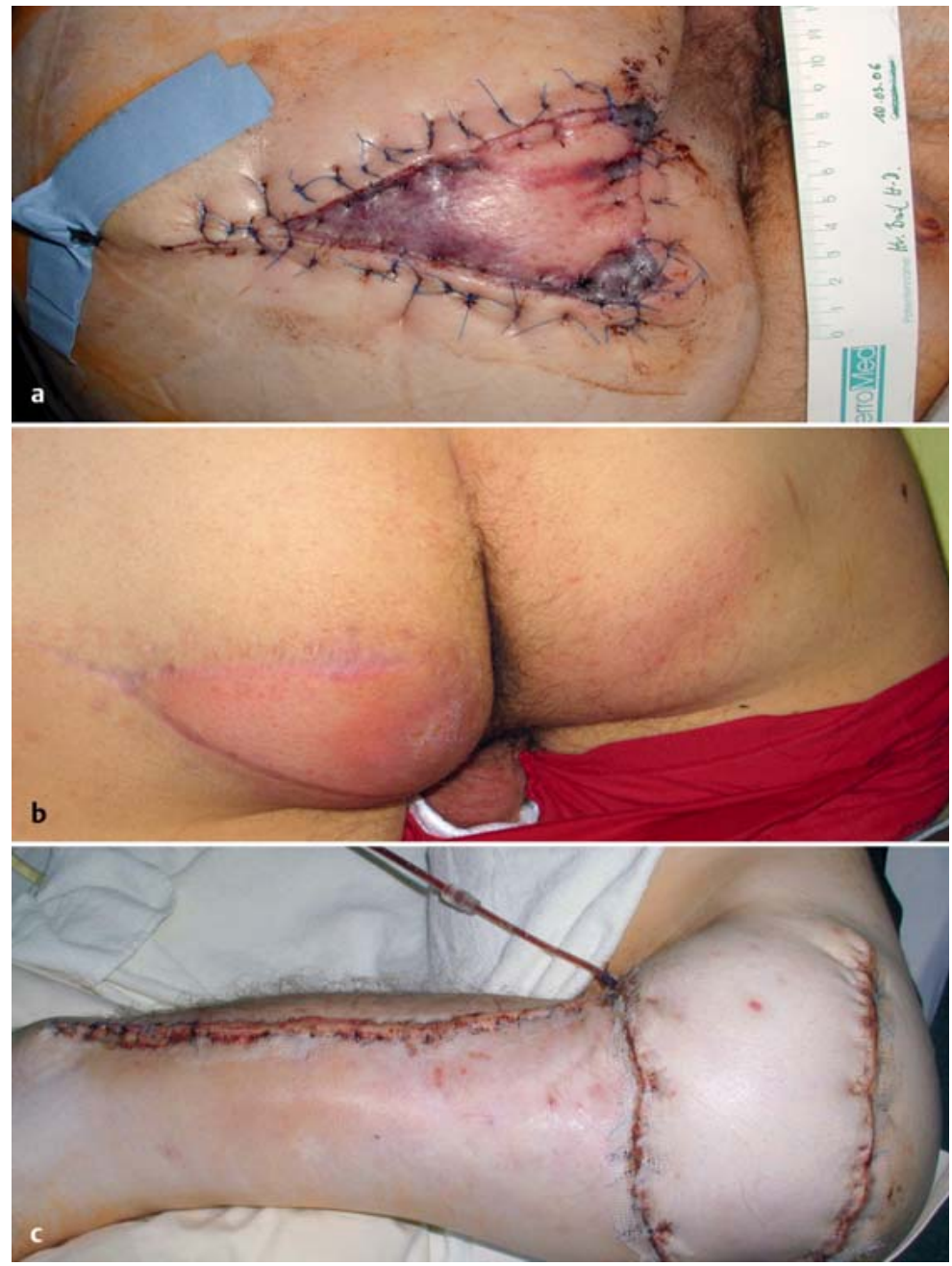

Abb. 10 - Komplikationen. a Frühkomplikation: Hämatom und Teilnekrose nach Schwenklappenplastik. b Spätkomplikation: Hämatom und Infektion bei zu früher Belastung. c Postoperatives Hämatom und Schwellung.
- Bei querschnittgelähmten Patienten mit aufgehobener Sensibilität sollte eine Anästhesie in Standby bereitgestellt werden, da diese Patienten Kreislaufinsuffizienzen, respiratorische Instabilitäten, autonome Massenreflexe und Muskelspasmen bieten können.

\section{Nachbehandlung}

- Konsequente Immobilisation mit optimaler Druckexposition postoperativ durch spezielle Lagerungstechniken und Lagerungsmatratzen, ggf. Wechseldruckmatratzen ist Grundvoraussetzung für den Erfolg einer Lappenplastik, da sonst Frührezidive drohen. Durch Spezialmatratzen ist z.B. eine Rückenlagerung auch bei sakralen Schwenklappenplastiken möglich.

- Eine Bauchlage sollte 10-14 Tage nach Lappenplastik im Gesäßbereich eingehalten werden. Für die psychisch belastende Bauchlage sind den Patienten sedierende Medikamente anzubieten.

- Nach 5-6 Wochen wird eine sukzessive Aufbelastung erfolgen.

- Eine Physiotherapie sollte insbesondere auch aus einer Atemgymnastik bestehen.

- Vor Entlassung aus dem Krankenhaus ist sicherzustellen, dass der Patient auf einer superweichen Matratze zu Hause liegen kann.

- Rollstuhlanpassung, Sitzkissenoptimierung, Schonung des Patienten.

\section{Komplikationen}

Lebensbedrohliche Komplikationen bestehen in der Ausbildung einer Sepsis oder Pneumonie. Durch eine entsprechende Prophylaxe kann die Inzidenz gesenkt werden (Abb. 10).

- Hämatome stellen die häufigste Komplikationsform. Diese sollten frühzeitig verhindert werden. Serome und nachfolgende Infektionen und Lappennekrosen begünstigen Rezidive mit Revisionen um 10-20\%.

- Serome entstehen auch durch ein zu geringes Lappenvolumen, inkomplette Resektion von Bursae und frühzeitige Druckbelastung des Schwenklappens. Drainagen sollten 5-10 Tage belassen werden.

- Wundheilungsstörungen treten in 15-30\% der Fälle auf.

- Instabile Narben können rezidivierend aufbrechen. Aus mehrfachen Rezidiven können selten Narbenkarzinome entstehen.

- Abhängig von der Lokalisation und Größe des Defekts entsteht ein Spenderarealdefekt mit teilweise Funktionsverlust z.B. der Muskulatur.

- Weitere Komplikationen sind Osteomyelitis, Bakteriämie, Sepsis. 


\section{Grenzen der chirurgisch-plastischen Dekubitusbehandlung}

In Grenzfällen ist ein Patient aufgrund allgemeiner Faktoren nicht operabel. Dies sind z. B. kardial oder pulmonal dekompensierte Patienten, ein schlecht eingestellter Diabetes mit starken Wundheilungsstörungen, Tumorkachexie usw. Trotz ausgedehnter Nekrosen kann eine aufwendige plastische Deckung nicht mehr ohne weitere Gefährdung des Patienten durchgeführt werden.

Der Erfolg einer Dekubitusbehandlung ist von weiteren Faktoren abhängig und kann nicht nur durch eine optimale konservative wie auch operative Therapie erreicht werden. Damit eine konservativ oder operativ behandelte Wunde heilen kann, braucht sie optimale Umgebungsvoraussetzungen. Hierzu sind die Vermeidung bzw. Verringerung von Risikofaktoren sowie wundheilungsfördernde Maßnahmen indiziert.

\section{- Behandlung von Risikofaktoren}

Primäre Risikofaktoren wie z.B. Tetraplegie oder Demenz können zwar nicht therapiert werden, jedoch können die sekundären Faktoren gezielt beeinflusst werden:

- Erhöhung des Sauerstoffangebots im Gewebe: Behandlung z.B. von Pneumonien, Herzinsuffizienz,

- Verbesserung des Sauerstofftransports, Nährstoffangebots und Verminderung des Sauerstoffverbrauchs: Nikotinabusus, Diabetes mellitus, Adipositas, chronische Erkrankungen des kardiovaskulären Systems.

\section{- Wundheilungsfördernde Maßnahmen und Vermeidung von Störfaktoren}

Beim Dekubitalulkus zeigt sich das typische klinische Bild einer gestörten Wundheilung. Vorrangiges Ziel ist die Wiederherstellung der physiologischen Wundverhältnisse, damit die Wundheilungskaskade beginnen und ablaufen kann. Wenn Wunden schlecht heilen, kann dies an Störfaktoren liegen, die vermindert bzw. vermieden werden müssen.

Mobilisation verbessert die Blutzirkulation, wodurch der Heilungsprozess initiiert und beschleunigt wird. Ein Bewegungsplan sollte sich an den individuellen Bedürfnissen des Patienten orientieren.

Als Primärfaktor gilt die Mangelernährung. Diese betrifft insbesondere ältere pflegeabhängige Patienten und jüngere Querschnittgelähmte mit Neigung zu einseitiger Ernährung.
Zur Diagnostik gehört ein Nutrigramm mit Überprüfung von Albumin, Transferrin, Zink, Vitamin $B_{12}$, Folsäure, Lymphozyten. Somit wird hinsichtlich der Ernährung bei Dekubitalläsionen eine ausgewogene Ernährung mit einer Energiezufuhr von 30-35 kcal pro kgKG und einer ausreichenden Proteinzufuhr von 1,0-1,5 g pro kgKG gefordert.

Zur Wundheilung benötigt der Körper Nährstoffe, die ggf. ersetzt werden müssen. Wirkung und Behandlung sind in (Tab. 3) aufgeführt. Weitere allgemeine und lokale Störfaktoren und ihre Behandlung zeigen Tab. 4 und Tab. 5.

\section{Tabelle 3}

\section{Nährstoffe und ihre Wirkung bei der Wundheilung.}

\begin{tabular}{|c|c|c|}
\hline Nährstoff & Rolle in der Wundheilung & Quellen \\
\hline Protein & $\begin{array}{l}\text { Kollagenbildung } \\
\text { Fibroblastenproliferation } \\
\text { Revaskularisation } \\
\text { Immunität } \\
\text { Bildung des lymphatischen Systems } \\
\text { Synthese und Sekretion von Wachsfaktoren }\end{array}$ & $\begin{array}{l}\text { Fleisch, Fisch, Eier, } \\
\text { Milchprodukte, Hülsenfrüchte, } \\
\text { Sojaprodukte, Getreide }\end{array}$ \\
\hline $\begin{array}{l}\text { Kohlen- } \\
\text { hydrate }\end{array}$ & Energie für Leukozyten und Fibroblasten & $\begin{array}{l}\text { Brot, Getreide, Nudeln, Reis, } \\
\text { Kartoffeln, Obst, Gemüse }\end{array}$ \\
\hline Fette & $\begin{array}{l}\text { Phospholipide der Zellmembran } \\
\text { Prostaglandinsynthese } \\
\text { Energiequelle }\end{array}$ & $\begin{array}{l}\text { pflanzliche Öle, Margarine, } \\
\text { Butter, Fischöle und versteckt } \\
\text { in fettreichen Lebensmitteln }\end{array}$ \\
\hline Vitamin A & $\begin{array}{l}\text { Cross-linking und „Remodeling“ } \\
\text { des Kollagens } \\
\text { Epthelialisierung } \\
\text { Immunantwort }\end{array}$ & $\begin{array}{l}\text { gelbe und grüne Gemüse } \\
\text { und Obst, Milchprodukte, } \\
\text { Eigelb, Leber, Lebertran }\end{array}$ \\
\hline B-Vitamine & $\begin{array}{l}\text { Verstoffwechselung von Proteinen, } \\
\text { Fetten und Kohlenhydraten }\end{array}$ & $\begin{array}{l}\text { Hefe, Leber, Fleisch, grüne } \\
\text { Gemüsesorten, Milch, } \\
\text { Vollkornprodukte }\end{array}$ \\
\hline Vitamin K & Synthese der Gerinnungsfaktoren & $\begin{array}{l}\text { Milchprodukte, Eigelb, Leber, } \\
\text { grüne Blattgemüse }\end{array}$ \\
\hline Vitamin C & $\begin{array}{l}\text { Hydroxylierung von Prolin und Lysin } \\
\text { in der Kollagensynthese } \\
\text { Leukozytenfunktion }\end{array}$ & Obst und Gemüse \\
\hline Zink & $\begin{array}{l}\text { Zellproliferation } \\
\text { Kofaktor der RNA- und DNA-Polymerase }\end{array}$ & $\begin{array}{l}\text { rotes Fleisch, Leber, Nüsse, } \\
\text { Vollkornprodukte }\end{array}$ \\
\hline Eisen & $\begin{array}{l}\text { Hydroxylierung von Prolin und Lysin } \\
\text { in der Kollagensynthese }\end{array}$ & $\begin{array}{l}\text { Fleisch, grünes Blattgemüse, } \\
\text { Vollkornprodukte }\end{array}$ \\
\hline
\end{tabular}




\section{Grundlagen}

Tabelle 4

Allgemeine Störfaktoren der Wundheilung und ihre Behandlung (nach Hartmann 2001).

\begin{tabular}{|c|c|c|c|}
\hline $\begin{array}{l}\text { Allgemeine Störfaktoren } \\
\text { der Wundheilung }\end{array}$ & Therapieoptionen & $\begin{array}{l}\text { Allgemeine Störfaktoren } \\
\text { der Wundheilung }\end{array}$ & Therapieoptionen \\
\hline $\begin{array}{l}\text { Infektionen } \\
\text { - Pneumonie (akut, chronisch) } \\
\text { - chronische Bronchitis } \\
\text { - Harnwegsinfektionen } \\
\text { (akut, chronisch) } \\
\text { - Osteomyelitis } \\
\text { - Sepsis } \\
\text { - Lokalinfektion des Ulkus } \\
\text { - Nekrose des Ulkus } \\
\text { - Fieber } \\
\text { - Leukozytose } \\
\text { - CRP-Anstieg } \\
\text { - Lymphopenie }\end{array}$ & $\begin{array}{l}\text { - Kausaltherapien, wenn immer } \\
\text { möglich, bzw. optimierte Therapien } \\
\text { der Grunderkrankungen } \\
\text { - Antibiotikatherapie } \\
\text { - Débridement } \\
\text { - Fieber senken } \\
\text { - optimale Ernährung }\end{array}$ & $\begin{array}{l}\text { Krankheiten } \\
\text { - Depression, Vereinsamung } \\
\text { - Anämie } \\
\text { - Dehydratation } \\
\text { - Diabetes mellitus } \\
\text { - Immunschwäche } \\
\text { - Herzinsuffizienz } \\
\text { - Niereninsuffizienz } \\
\text { - Krankheiten des Magen-Darm- } \\
\quad \text { Trakts } \\
\text { - Lähmungen } \\
\text { - Immobilität } \\
\text { - Nikotinabusus }\end{array}$ & $\begin{array}{l}\text { Krankheiten immer optimal } \\
\text { behandeln, da Krankheiten zum } \\
\text { Katabolismus führen } \\
\text { - Depression: SSRI, Betreuung } \\
\text { - Hämoglobin >11 g/dl } \\
\text { - Trinkmenge: > } 20 \mathrm{ml} / \mathrm{kgKG} \\
\text { - } \text { Diabetes mellitus: optimaler } \\
\text { Glukosespiegel } \\
\text { - Herzinsuffizienz, z. B. keine } \\
\text { Beinödeme! } \\
\text { - Lymphozytenzahl > } 2000 \text { (abs.) } \\
\text { - Serum-Zink > } 12 \mathrm{mmol} / \mathrm{l} \\
\text { - Mobilisieren }\end{array}$ \\
\hline $\begin{array}{l}\text { Malnutrition } \\
\text { - Katabolismus } \\
\text { - Appetitmangel } \\
\text { - Dehydratation } \\
\text { - eiweißarme Ernährung } \\
\text { - eiweißlose Ernährung } \\
\text { - fleischlose Ernährung } \\
\text { - Albuminmangel } \\
\text { - Transferrinmangel } \\
\text { - Ferritinmangel } \\
\text { - Cholinesterasemangel } \\
\text { - tiefes Cholesterin } \\
\text { - Vitamin-B12-Mangel } \\
\text { - Folsäuremangel } \\
\text { - Hyperhomocysteinämie } \\
\text { - Zinkmangel } \\
\text { - Eisenmangel } \\
\text { - Vitamin-D-Mangel }\end{array}$ & $\begin{array}{l}\text { Ursachen des Katabolismus suchen } \\
\text { - Ursache der Malnutrition suchen } \\
\text { (multifaktoriell), z. B. Magenulkus, } \\
\text { Depression, Zinkmangel } \\
\text { - Kausaltherapie der Malnutritionsursache } \\
\text { - optimale Ernährung: } \\
\text { - Proteine: } 1,0 \text { - } 1,5 \mathrm{~g} / \mathrm{kgKG} \\
\text { - Kalorien: } 30 \text { - } 50 \mathrm{kcal} / \mathrm{kgKG} \\
\text { - Fette: } 30 \% \text { der Tageskalorien } \\
\text { - Trinkmenge: }>20 \mathrm{ml} / \mathrm{kgKG} \\
\text { - Vitamin B12: } 10 \times 1 \mathrm{mg} \text { s.c. } \\
\text { - Folsäure } 1 \mathrm{mg} \text { per os } \\
\text { - Zink (org.) } 20 \mathrm{mg} / \text { Tag per os } \\
\text { - Eisen, immer intravenös, Dosis je nach } \\
\text { Schweregrad } \\
\text { - vollbilanzierte Trinknahrung bis } \\
\text { 1500 ml/Tag } \\
\text { - Multivitaminpräparat } \\
\text { - Kalzium-Vitamin-D-Präparat }\end{array}$ & $\begin{array}{l}\text { Medikamente } \\
\text { - Kortikosteroide } \\
\text { - sedierende Medikamente } \\
\text { - Zytostatika } \\
\text { - Immunsupressiva } \\
\text { - } \text { toxische Lokaltherapeutika, } \\
\text { z.B. Wasserstoffsuperoxyd }\end{array}$ & $\begin{array}{l}\text { - Lokaltherapeutika und Medika- } \\
\text { mente immer prüfen nach } \\
\text { negativen Effekten auf die } \\
\text { Wundheilung } \\
\text { - sedierende Medikamente } \\
\text { immobilisieren und erschweren } \\
\text { die Druckentlastung }\end{array}$ \\
\hline
\end{tabular}

Der Therapieerfolg lässt sich in Laborparametern wie CRP, Albumin, Lymphozyten und klinisch an der Bildung des Granulationsgewebes kontrollieren.

\section{Prognose}

Die Frage, ob ein Rezidiv bei optimaler Behandlung vermieden werden kann, ist mit „nein“ zu beantwortet. In der aktuellen Literatur wird das Rezidiv eines Dekubitalgeschwürs mit 30-60\% angegeben. Dies ist vor allem durch die Tatsache bedingt, dass durch eine plastische Operation das ursächliche Problem (z.B. Querschnitt- lähmung) nicht behoben werden kann. Somit ist es essenziell, eine suffiziente Rezidivprophylaxe mit Schulung der Patienten durchzuführen, die allerdings eine dementsprechende Compliance des Patienten voraussetzt. Wichtig ist in der postoperativen Phase die Ausschaltung der Noxe, in diesem Fall die Druckbelastung, wie die optimale technisch-orthopädische wie auch pflegerische Versorgung. 


\section{Tabelle 5}

Lokale Störfaktoren der Wundheilung und ihre Behandlung (nach Hartmann 2005).

\begin{tabular}{|c|c|}
\hline Lokale Störfaktoren (Lokalbefund) & Prozedere \\
\hline $\begin{array}{l}\text { ungenügende Druckentlastung } \\
\text { - weißlicher Wundrand } \\
\text { - sichtbare, 4-eckige oder runde Druckstempel } \\
\text { am Ulkusrand durch zu dicken Verband (>2 mm) }\end{array}$ & $\begin{array}{l}\text { - Druckentlastung optimieren } \\
\text { - nur dünne, feuchte Verbände verwenden }\end{array}$ \\
\hline $\begin{array}{l}\text { Nekrose } \\
\text { - schmierige Beläge } \\
\text { - schwarze Nekrosekruste } \\
\text { - Infektionsparameter erhöht }\end{array}$ & $\begin{array}{l}\text { - Débridement } \\
\text { - Druckentlastung optimieren } \\
\text { - nur dünne, feuchte Verbände verwenden } \\
\text { - Antibiotika systemisch, wenn Infektionsparameter erhöht }\end{array}$ \\
\hline $\begin{array}{l}\text { Lokalinfektion } \\
\text { - Ulkusrand: gerötet, überwärmt, ödematös, } \\
\text { druckschmerzhaft, schmerzend } \\
\text { - Infektionsparameter erhöht }\end{array}$ & $\begin{array}{l}\text { - Débridement } \\
\text { - permanentes Feuchthalten } \\
\text { - Verbandwechsel } 4 \times \text { täglich }\end{array}$ \\
\hline $\begin{array}{l}\text { Wundfläche ohne Granulation, ausgetrocknet } \\
\text { - Wundfläche ausgetrocknet } \\
\text { - Verband klebt } \\
\text { - nach Verbandwechsel: blutende Stellen, Gewebe klebt am } \\
\text { Verband; Schmerzen beim und nach dem Verbandwechsel }\end{array}$ & $\begin{array}{l}\text { - Wundspülung mit Ringer-Lösung } \\
\text { - permanentes Feuchthalten mit geeigneten hydroaktiven } \\
\text { Wundauflagen, z. B. Hydrokolloidverbänden }\end{array}$ \\
\hline $\begin{array}{l}\text { toxische Lokaltherapeutika; gewebetoxisch sind: } \\
\text { - Wasserstoffsuperoxyd } \\
\text { - starke Desinfektionsmittel } \\
\text { - gefärbte Lösungen usw. }\end{array}$ & - Wundmittel auf lokale Gewebetoxizität prüfen \\
\hline
\end{tabular}

\section{Begutachtung}

Eine lückenlose Dokumentation (auch bildtechnisch) des Behandlungsablaufs ist sinnvoll. Hierdurch lassen sich der Therapieerfolg messen und ggf. begründete Therapieregimeänderungen einleiten.

Gesetzlich ist der Nachweis einer dem aktuellen Standard entsprechenden ärztlich-pflegerischen Versorgung zur Pflicht erhoben, sodass die schriftliche Dokumentation auch zur (haftungs-)rechtlichen Absicherung der ärztlichen und pflegerischen Leistung unabdingbar ist.

\section{Perspektiven}

Die Entstehung und das Rezidiv eines Dekubitus sind häufig durch eine Prophylaxe vermeidbar.

Je besser jeder Patient aufgeklärt ist, desto besser kann die Entstehung eines Dekubitus vermieden oder, wenn vorhanden, effizienter behandelt werden (Tab. 6). Eine regelmäßige, adäquate Verlaufskontrolle und Wunddokumentation ermöglichen die zeitnahe Erfassung von Problemen und ein frühzeitiges Behandeln. Sollte es allerdings zur Entstehung eines Dekubitus gekommen sein, ist eine konsequente, stadiengerechte Therapie einzuleiten. Hierzu ist ein individuelles, patientenorientiertes, ganzheitliches und interdiziplinäres Vorgehen nötig. Es sollte alles unternommen werden, um die quälende und risikobehaftete Hospitalisationszeit für den Patienten zu verkürzen. Dies ist auch unter sozioökonomischer Sicht anzustreben. 


\section{Grundlagen}

Tabelle 6

Prävention von Dekubitalulzera (nach Anders et al. 2010).

\begin{tabular}{|c|c|c|c|}
\hline Präventive Prinzipien & Strategien & Präventive Maßnahmen* & Präventive Umsetzung \\
\hline \multirow[t]{7}{*}{ Risikominimierung } & Risikoerkennung & $\begin{array}{l}\text { Erfassung von Hochrisikogruppen (C) } \\
\text { regelmäßige Hautinspektionen (B) }\end{array}$ & $\begin{array}{l}\text { Einschätzung durch Fachkraft (Hautinspektion, } \\
\text { Mobilitätsstatus, Alter) Risikoskalen }\end{array}$ \\
\hline & Risikoeinschätzung & $\begin{array}{l}\text { Erfassung der begünstigenden } \\
\text { Risikofaktoren (C) }\end{array}$ & $\begin{array}{l}\text { Anamnese, Befunde, Hautinspektion geriatrisches } \\
\text { Assessment inklusive Behandlungsplan }\end{array}$ \\
\hline & Fehlervermeidung & Patientenidentifikation (C) & Kennzeichnung \\
\hline & & Dokumentation (C) & Lagerungsplan \\
\hline & & Kommunikation (C) & Pflegeüberleitung \\
\hline & & koordiniertes Handeln (C) & Teambesprechung \\
\hline & & Teamschulung (B) & Verfahrensanweisung \\
\hline \multirow[t]{12}{*}{ Druckentlastung } & Lagewechsel & individueller Lagerungsplan (C) & Frequenz durch Hautkontrollen bestimmen \\
\hline & Lagerungsarten & Umlagerung (A) & $30^{\circ}$-Schräglage \\
\hline & & Weichlagerung (A) & Rückenlage \\
\hline & & Fersenfreilagerung (B) & $135^{\circ}$-Lagerung \\
\hline & & Teamschulung (C) & Mikrolagerung \\
\hline & & & Langsitz \\
\hline & & & V-Lagerung \\
\hline & & & Nest-Lagerung \\
\hline & & & Hohl-Lagerung \\
\hline & $\begin{array}{l}\text { Knochenvorsprünge } \\
\text { entlasten }\end{array}$ & $\begin{array}{l}\text { direkten Kontakt von Knochen } \\
\text { auf Knochen vermeiden (C) }\end{array}$ & $\begin{array}{l}\text { versetzte Lagerung } \\
\text { Mikrolagerung }\end{array}$ \\
\hline & & & Unterpolsterung \\
\hline & Druckverteilung & $\begin{array}{l}\text { Lagerungssysteme (A) } \\
\text { individuelle Matratzenauswahl (A) }\end{array}$ & $\begin{array}{l}\text { Superweichmatratze (Auflagedruck ca. } 25 \mathrm{mmHg} \text { ) } \\
\text { oder Mikrostimulationssysteme, nur bei sehr } \\
\text { hohem Risiko oder zur Therapie (cave: mindert } \\
\text { Eigenwahrnehmung): Wechseldrucksysteme, } \\
\text { Luftbett (Low-Flow), nur bei Querschnittlähmung: } \\
\text { Drehbett, Sandwich-Bett }\end{array}$ \\
\hline \multirow[t]{2}{*}{$\begin{array}{l}\text { Vermeidung von } \\
\text { Hautverletzung }\end{array}$} & $\begin{array}{l}\text { Vermeidung von } \\
\text { Reibung und } \\
\text { Scherkräften }\end{array}$ & Lagerungstechnik (C) & $\begin{array}{l}\text { atraumatische Lagerung, Durchführung durch } \\
2 \text { Pflegekräfte, natürliche Schaffelle }\end{array}$ \\
\hline & & $\begin{array}{l}\text { Transfertechnik (C) } \\
\text { Hilfsmittel zur Fortbewegung (C) }\end{array}$ & $\begin{array}{l}\text { atraumatischer Transfer, natürliche Schaffelle, } \\
\text { En-bloc-Drehung, Durchführung durch } 2 \text { Pflege- } \\
\text { kräfte, Drehsitz, Ergotherapie }\end{array}$ \\
\hline \multirow[t]{2}{*}{$\begin{array}{l}\text { Vermeidung von } \\
\text { Mazeration }\end{array}$} & Hautschutz & Meidung von Nässe und Hitzestau (C) & $\begin{array}{l}\text { Hautschutzpflaster- oder -sprays (Acrylat- } \\
\text { Terpolymere, dünne Hydrokolloide), } \\
\text { regelmäßiger Wechsel von Inkontinenzhilfen }\end{array}$ \\
\hline & $\begin{array}{l}\text { Stärkung der } \\
\text { Hautbarriere }\end{array}$ & Hautpflege (B) & $\begin{array}{l}\text { Verwendung hautfreundlicher Syndets, } \\
\text { Hautpflege (Creme), Ernährung }\end{array}$ \\
\hline
\end{tabular}




\section{Tabelle 6}

Prävention von Dekubitalulzera (nach Anders et al. 2010).

\begin{tabular}{|c|c|c|c|}
\hline Präventive Prinzipien & Strategien & Präventive Maßnahmen* & Präventive Umsetzung \\
\hline \multirow[t]{2}{*}{ Bewegungsförderung } & passive Bewegung & $\begin{array}{l}\text { Lagerung (A) } \\
\text { Kontrakturprophylaxe (A) } \\
\text { sensorische Reize (C) }\end{array}$ & $\begin{array}{l}\text { Bewegung der Extremitäten und Gelenke, } \\
\text { basale Stimulation }\end{array}$ \\
\hline & aktive Bewegung & $\begin{array}{l}\text { Bewegungsanbahnung (A) } \\
\text { Bewegungsübung (A) }\end{array}$ & Physiotherapie, Training von Defiziten \\
\hline \multirow[t]{2}{*}{ Ernährung } & Flüssigkeit & Flüssigkeitszufuhr (C) & Trinkplan, parenterale Gaben \\
\hline & feste Nahrung & $\begin{array}{l}\text { Nährstoffzufuhr für alle } \\
\text { Dekubitusrisikopatienten (C) } \\
\text { Nährstoffzufuhr für Risikopatienten } \\
\text { für Mangelernährung, Multimorbi- } \\
\text { dität oder nach chirurgischen } \\
\text { Eingriffen (A) }\end{array}$ & $\begin{array}{l}\text { angereicherte Vollwertkost (hoch kalorisch, } \\
\text { proteinreich), supplementierte Nahrungs- } \\
\text { aufbereitung (z. B. Dysphagiekost), Nahrungs- } \\
\text { anreichung, parenteral }\end{array}$ \\
\hline
\end{tabular}

\section{Quellenangaben}

Anders J, Heinemann A, Leffmann C et al. Dekubitalgeschwüre Pathologie und Primärpravention. Dtsch Ärztebl 2010; 107: 371 - 382

Bruck JC. Dekubitalulzera: Algorithmus der Lappenplastiken. Plast Chir 2008; 4: $193-197$

Daniel RK, Hall EJ, MacLead MK. Pressure sorces - a reappraisal. Ann Plast Surg 1979; 3: 53-63

Die phasengerechte Wundbehandlung des Dekubitalulkus. Hartmann medicaledition 2005; 3: 2.3-78.79

Farhadi J, Pierer G. Plastisch-chirurgische Konzepte zur Deckung von Dekubitalulcera. Hartmann WundForum 2004; 3-4: 8-11

Flieger R, Meiners T. Dekubitus: Vermeidbare Komplikation neuromuskulärer Erkrankungen. Hefte zu „Medizinisch Orthopädische Technik“ 2005; 2: 13-24

von Goossens RHM, Zegers R, Hoek van Dijke GA et al. Influence of shear on skin oxygen tension. Clin Physiol 1994; 14: 111-118

Grundlagen zur Erarbeitung von Leitlinien zur Behandlung zur Dekubitusbehandlung. Hartmann WundForum 2001; 3: 27-33

Holle G, Peek A. Stellenwert der VAC-Therapie beim akuten und chronische Weichteiltrauma. Hefte zu „Medizinisch Orthopädische Technik“ 2008; 6: 49-56

Kremer M, Berger A. Plastisch-chirurgische Therapie beckennaher Dekubitalulzera. Chirurg. 1999; 70: 726-740

Kiss B. Die gestielte myokutane Gluteuslappenplastik - auch beim Rezidiv des präsakralen Dekubitus Methode der Wahl. Plast Chir 2008; 4 : 202-210

Schepler H. Gestielte und freie Perforatorlappen - Ein Paradigmenwandel in der Dekubituschirurgie. Plast Chir 2008; 4: 198-201
Turner TD. Hospital usage of absorbent dressings. Pharm J 1979; 222:

$$
421-426
$$

Young T. Pressure sores: incidence, risk assessment and prevention. Br J Nurs 1997; 6: 319-322

Zäch GA, Koch HG. Paraplegie, Ganzheitliche Rehabilitation. Basel: Karger; 2005: 209-221

\section{Korrespondenzadresse}

Dr. med. M. Schempf

Nova Clinic Biberach an der Riß

Eichendorffweg 5

88400 Biberach

Telefon: 0049-7351-44490

Fax: $\quad$ 0049-7351-444911

E-Mail: schempf@nova-clinic.de 


\section{Grundlagen}

\section{CME-Fragen}

Was sollte durch die VAC-Therapie nicht erreicht werden?

\section{CME.thieme.de}

\section{CME-Teilnahme}

- Viel Erfolg bei Ihrer CME-Teilnahme unter http://cme.thieme.de

- Diese Fortbildungseinheit ist 12 Monate online für eine CME-Teilnahme verfügbar.

- Sollten Sie Fragen zur Online-Teilnahme haben, unter http://cme.thieme.de/hilfe finden Sie eine ausführliche Anleitung.
Welcher Schwenklappen ist v.a. für die Os-sacrum-Region geeignet?
A Feuchte Wundreinigung.

B Reduktion von Wundödem bei kontinuierlichem Sog.

C Angiogenese.

D Reduktion der Bakterienlast.

E Druck auf das Ulkus.
Was ist die häufigste Komplikation nach Schwenklappenplastik?
A Tensor-fasciae-latae-Lappen.

B Bizepsschwenklappen.

C M.-gracilis-Lappen.

D Fasziokutaner VY-Lappen.

E Glutaeal Thigh Flap.
Bei welcher Begleiterscheinung eines Dekubitalulkus besteht eine absolute Operationsindikation?
A Drucknekrose.

B Kompartiment.

C Hämatome.

D Narbenkarzinome.

E Exsikkose.
A Schmerzen.

B Fistel.

C Sepsis.

D Aufwendige Pflege.

E Keine Möglichkeit der Druckentlastung.
Welche therapeutischen Maßnahmen gehören nicht zur konservativen Therapie eines Dekubitalulkus?
A Spezielle Lagerungskissen.

B Verbesserung des Gasaustauschs.

C Durch Verbände ein feuchtes Wundmilieu erhalten.

D Chirurgisches Débridement.

E Mikrolagerung. 
Was gehört nicht zur Prävention eines Dekubitalulkus?
A Regelmäßige Hautinspektion.
B Spezielle Lagerungssysteme.
C Anwendung von Transfertechniken.
D Allgemeiner Lagerungsplan.
E Ermittlung von Hochrisikogruppen.

Was gehört nicht zu den lokalen und allgemeinen Faktoren für die Wahl des Operationsverfahrens zur plastischen Deckung?
A Haut- und Weichteilzustand.
B Compliance des Patienten.
C Größe der operativen Einrichtung.
D Ulkusgröße und Tiefe.
E Allgemeinzustand des Patienten.
Welche Aussage trifft nicht zu?
A Sparsame Exzision des Ulkus, um das umliegende Gewebe maximal zu schonen.
B Die Nähte eines Schwenklappens sollten nicht in der Druckbelastungszone platziert werden.
C Der entstandene Totraum nach Ausschneiden eines Ulkus muss u. a. mit muskulären Lappenanteilen gedeckt werden.
D Anlegung einer Wunddrainage zur Hämatomvermeidung.
E Eröffnung des Periosts bei Osteomyelitis.

Welche Aussage trifft zu?

Welche Störfaktoren der Wundheilung und ihre Behandlung gehören nicht zusammen?

A Meistens handelt es sich bei Kontamination eines Ulkus um einen Keim, der gut mit einem spezifischen Antibiotikum therapiert werden kann.

B Bei offener Wundbehandlung ist generell eine antibiotische Therapie indiziert.

C Dekubitalulzera gelten primär mit Bakterien kontaminiert.

D Präoperative Darmreinigung ist nicht notwendig, da kein Zusammenhang zwischen Wunde und Darmausscheidung besteht.

E Bei Patienten mit einem Querschnittsyndrom muss keine Anästhesie bei Operationen unterhalb der Querschnittläsion bereitgestellt werden, da durch die Grunderkrankung das Schmerzempfinden aufgehoben ist.

A Lokalinfektion - Débridement.

B Verband klebt an der Wunde - langsames gewebeschonendes Abziehen des Verbands.

C Druckstempel am Ulkusrand - Druckentlastung.

D Schmierige Beläge im Wundgrund - Débridement.

E Wundfläche zu trocken - Hydrokolloidverbände. 